\title{
Optical tracing of multiple charges in single-electron devices
}

\author{
Sanli Faez, ${ }^{*}$ Sense Jan van der Molen, and Michel Orrit \\ Huygens-Kamerlingh Onnes Laboratorium, Universiteit Leiden, Postbus 9504, 2300 RA Leiden, The Netherlands \\ (Received 31 July 2014; revised manuscript received 20 October 2014; published 7 November 2014)
}

\begin{abstract}
Single molecules that exhibit narrow optical transitions at cryogenic temperatures can be used as local electricfield sensors. We derive the single-charge sensitivity of aromatic organic dye molecules, based on quantum mechanical considerations. Through numerical modeling, we demonstrate that by using currently available technologies it is possible to optically detect charging events in a granular network with a sensitivity better than $10^{-5} e / \sqrt{\mathrm{Hz}}$ and track positions of multiple electrons, simultaneously, with nanometer spatial resolution. Our results pave the way for minimally invasive optical inspection of electronic and spintronic nanodevices and building hybrid optoelectronic interfaces that function at both single-photon and single-electron levels.
\end{abstract}

DOI: 10.1103/PhysRevB.90.205405

PACS number(s): $32.60 .+\mathrm{i}, 42.50 . \mathrm{Ct}, 73.23 . \mathrm{Hk}$

\section{INTRODUCTION}

Sixty years after the invention of transistors, conduction electrons in solids are still the workhorses of information processing. Nanotechnology has enabled shrinking the size of transistors to a level where charge granularity and quantum effects emerge. Meanwhile, optical communication has widely replaced electronic communication because of its larger bandwidth and lower losses. With these developments comes the demand for an optoelectronic interface that operates at both single-electron and single-photon limits.

Single organic molecules in the solid state were first detected more than two decades ago by Moerner and Kador [1], using absorption spectroscopy, and by Orrit and Bernard [2] based on fluorescence emission. Since then, single-molecule fluorescence microscopy techniques have found a vast variety of applications in physics, chemistry, and life sciences. More specifically, in the past decade, there has been a surge in their applications in quantum optics. For example, organic molecules have proven to be ideal candidates for singlephoton sources [3]. Magnetic resonance of a single-electron spin has been demonstrated using spectroscopy on a single pentacene $[4,5]$. Other aromatic molecules have been used for quantum state preparation and readout [6], even at room temperature [7]. More recently, optical transistors were developed using the different energy levels of a single molecule [8] and coherent coupling between distant molecules has been achieved [9].

Empowered by these accomplishments, we can now consider single molecules as building blocks for integrated photonics circuits and hybrid quantum devices [10]. Successful insertion of these molecules into nanochannels, without any degradation of their coherence properties, has already allowed for demonstration of coherent interaction of light with several molecules in a dielectric waveguide [11]. This result has also paved the way for on-demand insertion of organic molecules in other electronic nanodevices as quantum nanoprobes.

In this paper, we demonstrate that single organic molecules can be used for optical detection and locating individual electrons. At cryogenic conditions the optical transition linewidth of some aromatic molecules in solid host matrices is narrower

*Faez@physics.leidenuniv.nl than $30 \mathrm{MHz}$ [12]. We illustrate how the movement of a single electron in the micrometer surrounding of such a molecule would change its transition frequency by several times its linewidth. As a result, the electron can be optically traced by following the molecular line shift. To demonstrate the speed and sensitivity of this sensing method, we consider a generic single-electron device that consists of a single metallic island and two electrodes. Further, we present our simulation results on feasibility of locating the position of multiple electrons with nanometer spatial resolution. These simulations are implemented considering a nanoparticle chain that exhibits Coulomb blockade as the simplest relevant example. All suggested measurements can be performed based on currently available knowledge and technology.

\section{A. Molecular nanoprobes}

At moderately low temperatures, around $2 \mathrm{~K}$, certain organic molecules present a very strong zero-phonon line (ZPL) on their electronic transition at optical frequencies (500 THz for visible red light) with a lifetime-limited linewidth of less than $30 \mathrm{MHz}$ [12]. The nominal quality factor corresponding to this resonance is better than $10^{7}$. As such, each molecule can be seen as a highly sensitive local probe of its environment. Experiments have shown that some of these molecules can acquire a dipole moment difference as large as 1 Debye $\left(3.3 \times 10^{-30} \mathrm{C} \cdot \mathrm{m}\right)$ between their electronic ground and excited states [13]. The transition frequency is thus very sensitive to the local electric field. For example, a sizable fraction of terrylene molecules in a $p$-terphenyl crystal exhibit a linear line shift of more than $3 \mathrm{MHz} /(\mathrm{kV} / \mathrm{m})$ in response to external electric field [14], despite the centrosymmetry of the terrylene molecule. This anomalously high linear response is induced by the deformation of the molecular orbital in the crystal [15]. The electrostatic field of a single electron at a $100 \mathrm{~nm}$ distance is roughly $150 \mathrm{kV} / \mathrm{m}$. This field is high enough to shift the transition frequency of these molecules by more than three times its $42 \mathrm{MHz}$ linewidth [16]. Considering this gigantic sensitivity and the small size of these molecules, Caruge and Orrit have suggested to detect electronic currents in semiconductors using organic molecules as nanoprobes [17]. Later, Plakhotnik has shown that subnanometer displacement of a single electron in a dielectric medium can be detected by simultaneously looking at the line shifts of multiple molecular 
probes $[18,19]$. Here, we will use a typical electron-in-a-box model and the Fermi golden rule for spontaneous emission to identify the origin of this sensitivity and routes to its optimization.

\section{THEORY}

The common feature in the conjugated molecules that show lifetime-limited linewidth is their rigid backbone. Quantum mechanical calculations based on box boundary conditions have been relatively successful in predicting the optical transition frequencies of this type of molecules [20]. For our discussion, considering the simplest model of an electron in a one-dimensional box is sufficient. The eigenfunctions of this model correspond to molecular $\pi$ orbitals that are filled with a total number or $N$ electrons. The transition frequency from the highest occupied molecular orbital (HOMO) to the lowest unoccupied molecular orbital (LUMO) is given by

$$
v=\frac{(N+1) h}{8 m_{e} L_{M}^{2}},
$$

where $h$ is the Planck constant, $m_{e}$ is the electron mass, and $L_{M} \equiv A_{M} a_{0}$ is the nominal length of the box. We take the Bohr radius $a_{0}=52.9(2) \mathrm{pm}$ as the smallest relevant length scale for defining molecular dimensions. The numerical factor $A_{M}$ can be chosen such that the values of the optical transition in the model match the experimental result. It helps our discussion to reformulate Eq. (1) as

$$
L_{M} v=\frac{\pi(N+1) c \alpha}{4 A_{M}},
$$

with $c$ the speed of light in vacuum and $\alpha \equiv \frac{h}{2 \pi m_{e} c a_{0}}$ the fine structure constant. The frequency width of the transition is given by the Fermi golden rule, considering nonradiative decay is negligible:

$$
\Gamma_{0}=\frac{8 \pi^{2} n_{h} d_{M}^{2} \nu^{3}}{3 \epsilon_{0} h c^{3}} .
$$

The refractive index of the medium at frequency $v$ and the vacuum permittivity are denoted by $n_{h}$ and $\epsilon_{0}$, and $d_{M}$ is the transition dipole moment. This dipole moment can be written as

$$
d_{M}=B_{M} e L_{M},
$$

where $e$ is the elementary charge and $B_{M}$ is a system-dependent numerical prefactor, which can be calculated based on either first principles or experimental results. Dividing Eq. (2) by Eq. (3) yields the nominal quality factor of the molecular transition

$$
Q \equiv \frac{v}{\Gamma_{0}}=\frac{3 A_{M}^{2}}{\pi^{4}(N+1)^{2} n_{h} B_{M}^{2}}\left(\frac{1}{\alpha}\right)^{3} .
$$

The system dependent prefactor on the right hand side of Eq. (5) happens to be in the order of unity for the aromatic molecules that are relevant to the subject of this paper. For the exemplary system of terrylene in p-terphenyel [14], this prefactor is 4.8 . The narrow linewidth of the molecular transitions, with high quality factors of $Q=10^{7}$, have a wellknown connection with the small value of the fine structure constant $[21,22]$.
We proceed with the calculation of the line shift in response to external electric field. In general, this Stark shift can be written as

$$
h g_{\mathrm{S}}=-\vec{\mu}_{M} \cdot \mathbf{E}(\mathbf{r})-\frac{1}{2} \mathbf{E}(\mathbf{r}) \cdot \overline{\bar{\Theta}}_{M} \cdot \mathbf{E}(\mathbf{r}),
$$

where $\vec{\mu}_{M}$ and $\overline{\bar{\Theta}}_{M}$ are the changes in the molecular dipole moment and molecular polarizability tensor upon excitation. $\mathbf{E}(\mathbf{r})$ is the local (quasi)static electric field at the position of the molecule. The local field is mainly determined by the immediate surrounding of the molecule. In common single-molecule spectroscopy measurement, the environment configuration is frozen, hence the changes in the transition energy can still be related directly to the external field provided that the local field effects are properly included [23]. Implementing this correction does not influence the present discussion. For the purpose of electric-field sensing, we are interested in large Stark shifts, which are mainly found for emitters with broken centrosymmetry, either internally or induced by the host matrix. In this case, the linear term in Eq. (6) dominates. Similar to the transition dipole moment, we relate the linear dipole change to the elementary charge and the nominal length of the molecule by

$$
\mu_{M} \equiv\left|\vec{\mu}_{M}\right|=C_{M} e L_{M}
$$

The change of electric field due to small radial displacement $\Delta r$ of a single-electron charge at a distance $r$ from the molecule is given by

$$
\Delta E(r)=\frac{1}{2 \pi \epsilon \epsilon_{0}}\left(\frac{\Delta r}{r^{3}}\right),
$$

with $\epsilon$ the static dielectric constant. To define a figure of merit for sensitivity, the Stark shift due to this field variation should be compared with the natural linewidth. By inserting Eq. (8) in Eq. (6) and dividing it by Eq. (3) and considering the emission wavelength in vacuum $\lambda=c / \nu$, we obtain

$$
\frac{g_{\mathrm{S}}}{\Gamma_{0}}=\frac{3 C_{M}}{16 \pi^{3} \epsilon n_{h} B_{M}^{2}}\left(\frac{\lambda}{r}\right)^{3} \frac{|\Delta r|}{L_{M}} .
$$

This equation is the key result of this discussion. Apart from the numerical prefactor, this general argument holds for any single-electron two-level emitter that decays solely by spontaneous emission and is protected from other sources of decoherence like for example nitrogen vacancy centers in diamond [24]. Although modern spectroscopy techniques enable us to measure line shifts much smaller than a linewidth, we choose to be conservative and call a nanoprobe sensitive when the coupling factor $g_{S} / \Gamma_{0}>1$. Equation (9) shows that such an emitter is sensitive to the movement of a single elementary charge within its optical near-field volume. This volume, set by the radiation wavelength, is generally much larger than the emitter size. The same interpretation, for example, clarifies the sensitivity of a superconductive Josephson qubit, with a microwave transition, to charge fluctuations in its extended environment [25]. In Table I, we list the experimentally measured properties of several aromatic molecules that exhibit, under cryogenic conditions, both a lifetime-limited ZPL and a large linear response to external electric field. 
TABLE I. Summary of optical transition properties for dibenzoterylene (DBT), dibenzoanthranthene (DBATT), terrylene (Ter) in two host matrices, as well as CdSe colloidal nanocrystals for comparison.

\begin{tabular}{lccccc}
\hline \hline Emitter/Host & $N$ & $\lambda(\mathrm{nm})$ & $\Gamma_{0}(\mathrm{MHz})$ & $\mu_{M} / e(\mathrm{pm})^{\mathrm{a}}$ & Reference \\
\hline DBT/anthracene & 38 & 784 & 30 & 1.2 & {$[38]$} \\
DBATT/hexadecane & 30 & 589 & 17 & 6 & {$[39]$} \\
Ter/p-terphenyl & 30 & 579 & 42 & 6 & {$[14]$} \\
Ter/polyethylene & 30 & 575 & 42 & 10 & {$[13]$} \\
CdSe/- & - & 620 & $10^{6 \mathrm{~b}}$ & 100 & {$[40,41]$} \\
\hline
\end{tabular}

${ }^{a}$ Typical value at the shoulder of the inhomogeneous distribution.

${ }^{b}$ Experimental value, broadened by spectral diffusion.

\section{NUMERICAL RESULTS}

The remarkable sensitivity of molecular nanoprobes to the presence of charges in their extended surrounding allows for tracing conduction electrons in solid-state systems. In the following, we use numerical modeling to demonstrate the working principles of this method, its time resolution, and its spatial accuracy. For this purpose, we consider the most generic geometries that are commonly used for modeling single-electron studies. We assume a planar device made of flat conducting electrodes and metallic islands in between them. The organic host crystal is doped with the aromatic molecules and put on top of the devices. This layer is separated by a thin insulating layer that covers the electronic device to eliminate possibility of charge transfer between the device and the crystal. As molecular nanoprobe we take the system of terrylene in $p$-terphenyel due to its superior brightness and spectral stability. The zero-phonon transition emits at $\lambda=$ $579 \mathrm{~nm}$ with a linewidth of $\Gamma_{0}=42 \mathrm{MHz}$ and an experimentally measured Stark coefficient of up to $3 \mathrm{MHz} /(\mathrm{kV} / \mathrm{m})$ [14].

\section{A. Single-electron box}

The simplest electronic device that exhibits single charge transport is the electron box. It consists of a nanoscale metallic island capacitively coupled to junction electrodes. We consider first the regime of large tunneling resistivity so that the charging and discharging times $t=R C$ are larger than the required measurement time of about a few milliseconds. Here, $R$ and $C$ are the total resistance and the total capacitance, respectively, between the island and the rest of the system. To show the sensitivity as a function of molecule-island separation, we consider addition of a single charge to the island while the electrodes are connected to ground. This will allow us to investigate the effect of field screening by the junctions, For simplicity, we model them by small grounded spheres. The capacitance between the island and each junction is denoted by $C_{j}$ and the gate capacitance is $C_{g}$. Considering this simple geometry, the position dependence of molecule-electron coupling constant $g_{\mathrm{S}} / \Gamma_{0}$, i.e., the line shifts of single terrylene molecules after adding one electron to the island, are calculated based on the electrostatic field distribution and the experimentally determined values of Stark coefficient and linewidth.

The results are depicted in Figs. 1(a) and 1(b) for dipole change orientation parallel and perpendicular to the substrate,
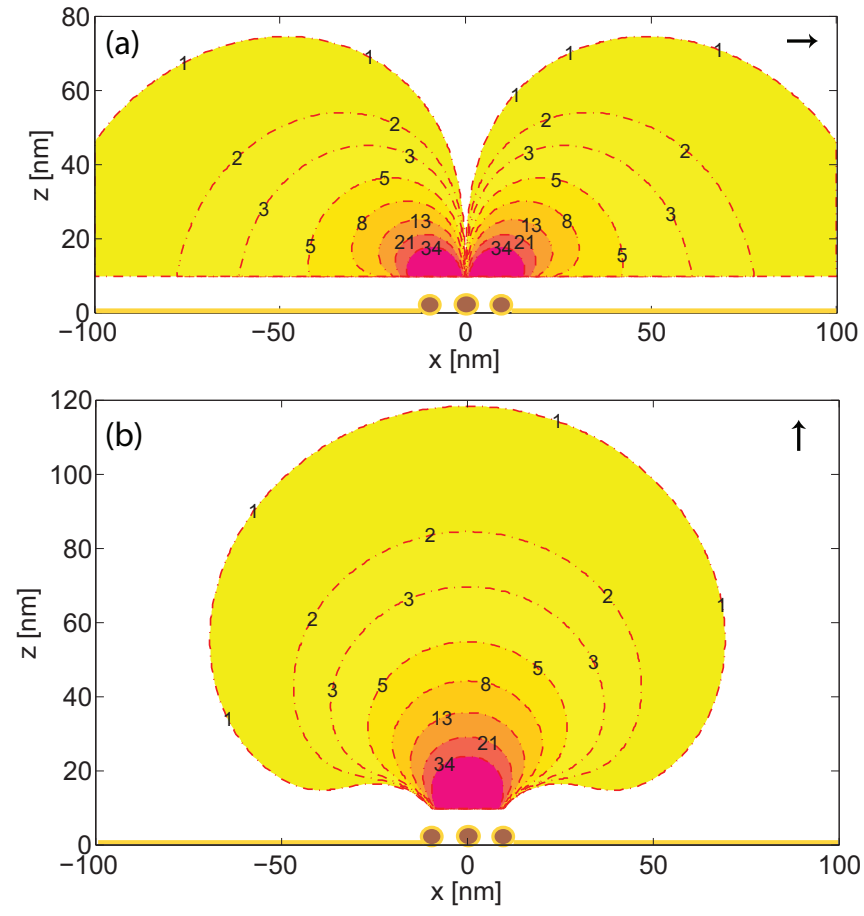

FIG. 1. (Color online) Contour plot of the coupling constant $\left(\left|g_{\mathrm{S}} / \Gamma_{0}\right|\right)$ as a function position above a single-charged island between grounded electrodes for (a) parallel and (b) perpendicular dipole change orientations of the molecules with respect to the substrate. Parameters: $\Gamma_{0}=42 \mathrm{MHz}, \mu_{M} / e=6 \mathrm{pm}, \epsilon=2.5$, $C_{g} / C_{j}=3$.

respectively. This plot demonstrates the superior charge sensitivity of a single organic molecule. We find that the transition of a molecule located $10 \mathrm{~nm}$ away from the island reacts to a charge difference of $10^{-2} e$ by a full linewidth shift, hence is no longer excited at the initial excitation frequency. The total emission rate of the molecule is limited by its upper-state lifetime of a few nanoseconds. In practice, due to the limited collection efficiency, it is possible to detect around $10^{6}$ photons per second on a single-photon detector without any significant line-broadening [26]. With these figures, the charge sensitivity of such an optical detection can easily reach $10^{-5} e / \sqrt{\mathrm{Hz}}$, which is on par with the highly celebrated sensitivity of single-electron transistors [27]. This is just an empirical value for the simplest configuration presented here.

\section{B. Localization accuracy}

Next to sensing the presence of charges on a metallic island, molecular nanoprobes can also be used to localize the position of electrons in a granular network. In cryogenic single-molecule spectroscopy, molecules are distinguished through their ultranarrow spectral response and hence they can be localized with an accuracy far beyond the diffraction limit [28]. Unlike room-temperature localization microscopy, cryogenic spectroscopy measurements can handle molecular concentrations as high as $10^{4}$ per cubic micrometer. Furthermore, given the unlimited photon budget, the position of each molecule can be determined with an accuracy better than one nanometer [29,30]. 
To determine the coordinates of a single electron in three dimensions, the simultaneous line shift of three or more molecules can be used. This triangulation method and its accuracy have been previously discussed in the context of studying charge transfer in a chemical bond [18]. In that work, an optimal localization precision of about $10 \mathrm{pm}$ has been suggested based on Monte Carlo simulations. Here, we present a more comprehensive analysis, considering the experimentally relevant parameters, for the goal of imaging distribution of multiple charges and their dynamics in electronic nanodevices.

\section{Nanoparticle chains}

To visualize parallel detection of several conduction electrons, we take a nanoparticle chain device at cryogenic temperatures as a generic example. This and other granular systems have been in use from the early days of measuring single-charge transport [31], but are still the topic of active research in the context of molecular electronics [32,33]. For clarity of presentation, we restrict our discussion to the case of a linear chain of capacitively coupled small islands. We note that the same procedure can be applied to two-dimensional network of particles and our conclusions are not restricted to a linear chain. For identifying the position of $n$ electrons on such a linear chain, in the most general case, there are $n+1$ nanoprobes necessary. The main sources of inaccuracy are the localization error of the molecules and uncertainties in their Stark factors. To increase the accuracy, it is necessary to probe a larger number of molecules than electrons and thereafter solve an over-parameterized inverse problem. These molecules are distinguished both in position and in excitation frequency and following the spectral shifts of several molecules, simultaneously, is straightforward.

We then perform Monte Carlo simulations with synthetic errors to estimate the propagating errors due the positioning inaccuracy. The modeling scheme is as follows. We consider 10 probe molecules evenly spaced on a line parallel to the substrate with a intermolecular separation of $20 \mathrm{~nm}$. The spacing between the molecules and the substrate is also set to $20 \mathrm{~nm}$ as schematically presented in Fig. 2(a). A total number of $n<10$ electrons are randomly distributed inside a $200 \mathrm{~nm}$ interval on the substrate and the induced molecular line shifts relative to the charge-neutral state are calculated. The experimental uncertainty is simulated by imposing \pm 1 $\mathrm{nm}$ localization errors on the lateral position of the molecules, varying their separation from the substrate within $\pm 3 \mathrm{~nm}$, and varying the magnitude of the shifts by $\pm 3 \%$. One example is plotted in Fig. 2(b). Note that although these localization inaccuracies are much smaller than the optical diffraction limit, they are now routinely accessible in cryogenic conditions using single-molecule localization techniques [30]. The positions of electrons or, strictly-speaking, the metallic grains that are charged by these electrons, are recalculated based on a least-square optimization routine. The mean deviation is estimated by taking the average of the absolute difference between the reconstructed and actual positions of the electrons for many realizations. As an example, reconstructed positions are plotted versus the actual positions for ten realizations of $n=6$ electrons in Fig. 2(c). This plot, highlights the high accuracy of locating multiple electrons simultaneously based (a)
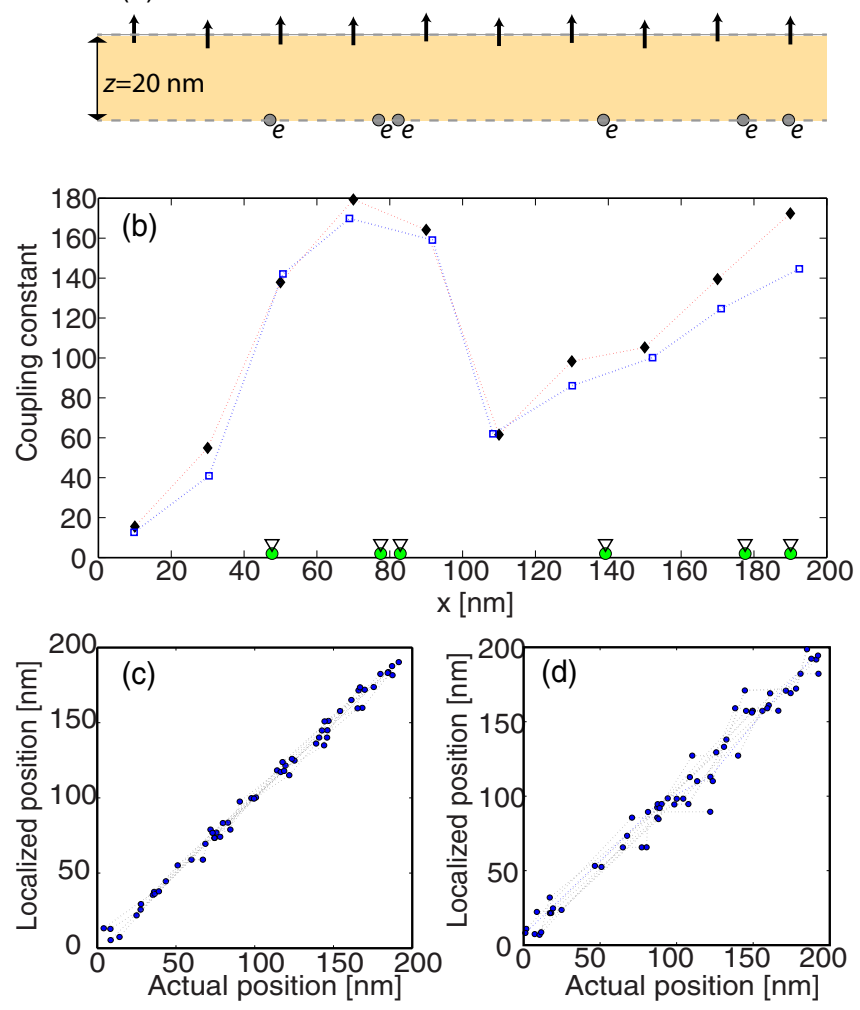

FIG. 2. (Color online) (a) The modeling procedure. Probe molecules, depicted by arrows, are evenly separated at an average spacing of $20 \mathrm{~nm}$ from each other on the $z=20 \mathrm{~nm}$ line. All molecules are aligned and respond maximally to electric field in the $z$ direction. Charges are randomly distributed on the $z=0$ line. The positions for a typical realization with $n=6$ electrons are indicated by circles. (b) The corresponding line shifts relative to the linewidth of the molecules, induced by the presence of electrons, are indicated by squares. The values for the line shifts are changed randomly by \pm 3 percent of the actual values and the molecule coordinates are changed by adding a random displacement in both $x$ and $z$ directions. The new values for this specific realization are indicated by filled diamonds, from which the charge distribution is reconstructed (downward arrows close to the lower axis). Parameters: ten molecules, six electrons, $\Gamma_{0}=42 \mathrm{MHz}, \mu_{M} / e=6 \mathrm{pm}, \epsilon=2.5$. (c) The localized positions of six electrons in ten random configuration compared with the actual positions considering a localization inaccuracy of $\pm 1 \mathrm{~nm}$ in the $x$ direction and $\pm 3 \mathrm{~nm}$ in the $z$ direction. The six data points corresponding to each realization are connected by dotted lines for clarity. (d) Similar to (b) but considering an exaggerated localization inaccuracy of $\pm 10 \mathrm{~nm}$ in both $x$ and $z$ directions.

on our suggested technique. To emphasize on the robustness of this method, we have also followed the similar reconstruction routine considering an exaggerated localization inaccuracy of $\pm 10 \mathrm{~nm}$ in both $x$ and $z$ directions and the results are depicted in Fig. 2(d).

The summary of simulation results are plotted in Fig. 3 as a function of $n$. For each point in the plot, 300 realization are simulated. These results show a localization accuracy of $1.5 \mathrm{~nm}$ when a single electron is probed using ten molecules. Most notably, this value is even smaller than the optical localization inaccuracy that we considered for each 

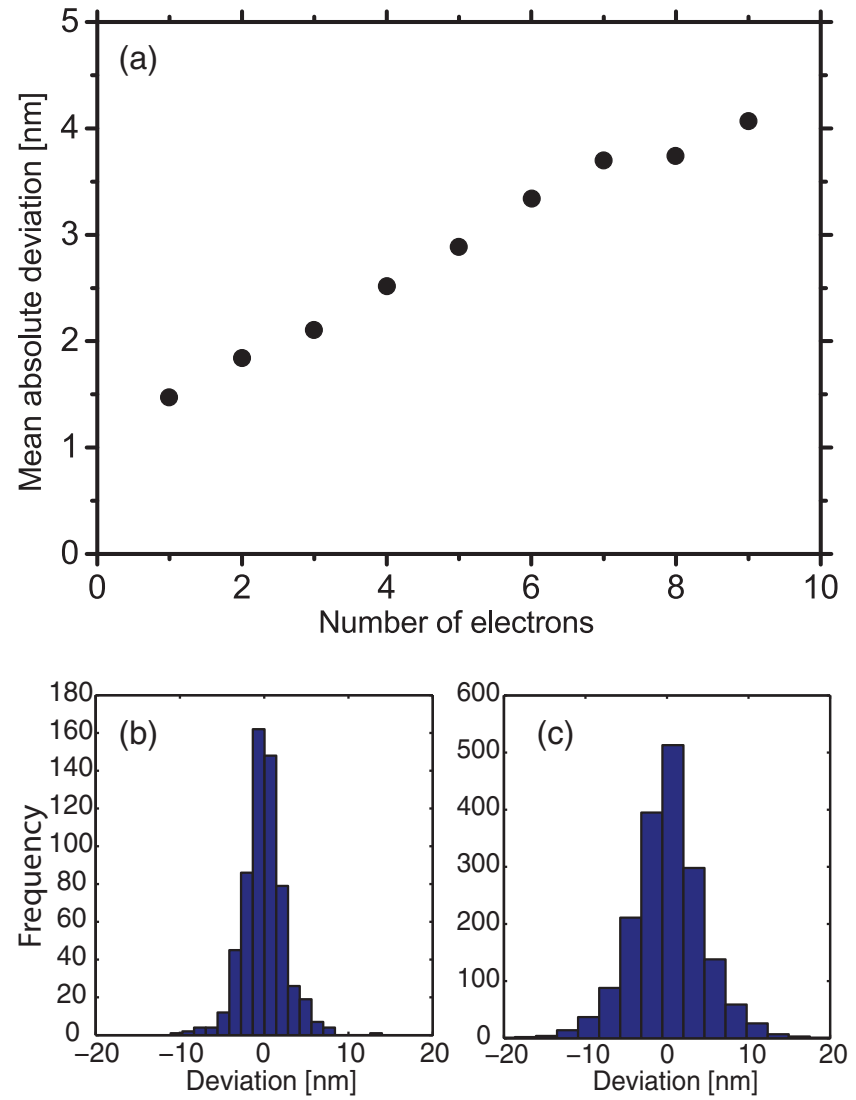

FIG. 3. (Color online) (a) The reconstruction inaccuracy for $n$ electrons when using ten probe molecules. (b) The deviation histogram for $n=2$ based on 300 realizations. (c) Same for $n=6$. Simulation parameters are the same as in Fig. 2(b).

individual molecule. The mean deviation between localized and actual positions increases monotonically with the number of electrons that are simultaneously probed, while keeping the number of probe molecules constant. This result proves the scalability of our suggested technique for parallel tracing of multiple electrons.

We also consider the case where the actual number of charges on the nanoparticle chain is treated as an unknown variable for the optimization procedure. For this problem, we repeat the optimization routine, assuming a different number of electrons $n^{\prime}$ in each run. The mean residual of each fit is plotted as a function of $n^{\prime}$. This value defined as the norm of the residuals vector for the best fit divided by the number of probe molecules. The position of minimum in this plot indicates the best guess for the actual number of charges in the chain. An example is shown in Fig. 4 for the case $n=6$ charges on the network and imposing the same accuracies in positions and line shifts as before. This plot shows a clear minimum at $n^{\prime}=6$ corresponding to the actual number of charges used for simulating the line shifts. From this practice, we can conclude that mapping multiple charges with the proposed method of this paper is also feasible without prior knowledge of their exact number.

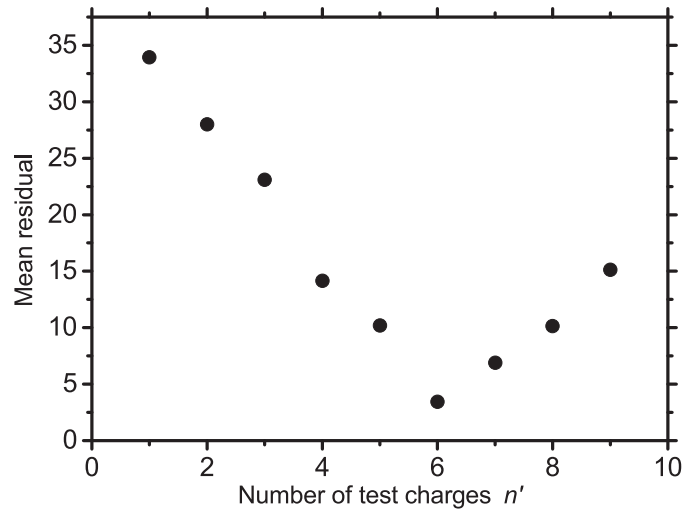

FIG. 4. Mean residual as a function of assumed number of test charges used for reconstructing the simulated line shift caused by an actual number of $n=6$ electrons. Simulation parameters are the same as in Fig. 2(b).

\section{Planar nanoparticle networks}

In principle, considering a sufficient number of probe molecules, the position of a single point charge in a dielectric can be localized in all three dimensions. When considering a metallic granular system, the inside charges will be screened. The position of the surface charges, however, can still be detected by an algorithm similar to the previous section. To demonstrate the feasibility of charge mapping in more than one dimension, we consider a a planar metal nanoparticle network $[31,33]$. This system and its variants are broadly used for studying the electronic transport in nanostructured devices.

Similar to the simulation for a linear chain, we consider the probe molecules all lie on a plane parallel to the device at a fixed distance. This realization is sketched in Fig. 5(a). We have already discussed the dependence of localization error on the number of charges in the previous section. Therefore, for this two-dimensional geometry, we only consider simultaneous localization of two electrons by using nine probes. The measurements errors are also imposed in the same way as in Sec. III C. The simulation results for the deviation between localized and actual position of the electrons are presented in Figs. 5(b) and 5(c). We find a median localization error of around $2 \mathrm{~nm}$, which is comparable to the result for the linear chain.

\section{DISCUSSION}

Although electronic amplifiers allow routine measurement of minuscule currents nowadays, several of these techniques fail in the total Coulomb blockade regime where current is vanishingly small. Some scanning methods such as Kelvin probe microscopy [34] also function based on static charge mapping. However, scanning methods like this are inherently slow and currently incapable of addressing multiple locations in parallel. In our technique, as demonstrated above, several molecules in the 500-nm surrounding of a device can act as simultaneous probes. This allows parallel mapping of charge distribution with an information density and speed that is unsustainable for scanning probe techniques.

We emphasize that our choice of a simple single-electron device was for demonstrating the working principles of this 
(a)

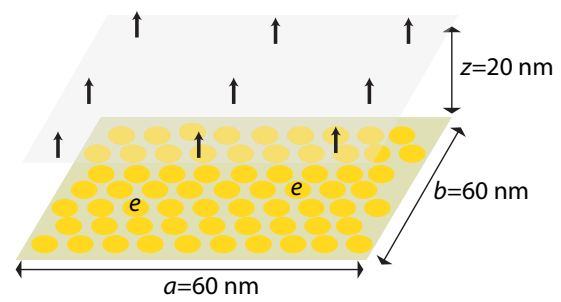

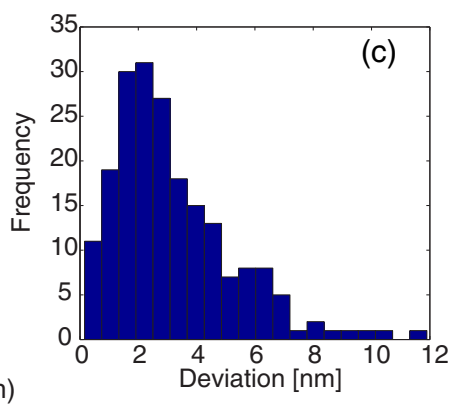

FIG. 5. (Color online) (a) Localizing electrons on a planar network. Probe molecules, depicted by arrows, are evenly separated at an average spacing of $20 \mathrm{~nm}$ from their nearest neighbors on the $z=20 \mathrm{~nm}$ plane above the nanoparticle device. All molecules are aligned and respond maximally to electric field in the $z$ direction. Point charges are randomly distributed on the $z=0$ plane. The simulation parameters are the same as in Fig. 2. (b) The deviation of reconstructed charges from the actual position of $n=2$ electrons in 100 random configuration, considering a molecule localization inaccuracy of $\pm 1 \mathrm{~nm}$ in the $x$ and $y$ directions and $\pm 3 \mathrm{~nm}$ in the $z$ direction. (c) Histogram of the absolute value of the deviation for the data points plotted in (b).

minimally invasive optical tracing technique. As we have discussed, the main strengths of using molecular nanoprobes for tracing charges, in comparison with conventional electrometrical techniques, is in visualizing the slow dynamics of multiple static charges. However, this method can also be extended, by fast and shot-noise limited photon detection, to measure the full counting statistics of charge transport [35], which is a more general form of current measurement. The mean current can then be determined from the peak of the charge fluctuations power spectrum [36]. This is somehow similar to the correlation measurements that are commonly used to quantify for example the triplet-state lifetime in molecules or other sources of spectral diffusion [37]. Here, the fluorescence signal from a molecule is measured by time-correlated photon counting, while the frequency of the excitation laser is fixed. Given the brightness of these molecules, this time trace can be recorded at $10-\mathrm{MHz}$ rate with an event time accuracy of $100 \mathrm{ps}$. In the charging interval between successive tunneling events, the molecule line is shifted and hence it will be dark. The rate of charging events and its duration can be recovered from the autocorrelation of the fluorescence time trace.

In conclusion, we have presented an optical method for measuring static charge distribution in electronic devices such as single-electron transistors or granular networks and have demonstrated its sensitivity. Our approach is an alternative to scanning probe techniques, such as Kelvin probe microscopy, for investigation of charge distribution in solid state nanodevices. Using numerical simulations, we have illustrated a spatial accuracy of $1 \mathrm{~nm}$ for positioning a single electron and less than $3 \mathrm{~nm}$ for tracking of up to six electrons simultaneously on a nanoparticle chain, using ten probe molecules. Similar simulations for positioning of two electrons in a planar network with nine probe molecules result in a median inaccuracy of $2 \mathrm{~nm}$. The required organic substances are soft and easy-tohandle materials, and can be placed on top of many other conducting or semiconductor devices with minimum difficulty. Since our electron sensing method functions at single-photon, single-molecule, and single-electron levels, it sets the basis for building hybrid quantum devices.

\section{ACKNOWLEDGMENTS}

This research was supported by the Nederlandse Organisatie voor Wetenschappelijk Onderzoek, NWO (Vidi grant SJvdM), and European Research Council (Advanced Grant SiMoSoMa).
[1] W. E. Moerner and L. Kador, Phys. Rev. Lett. 62, 2535 (1989).

[2] M. Orrit and J. Bernard, Phys. Rev. Lett. 65, 2716 (1990).

[3] C. Brunel, B. Lounis, P. Tamarat, and M. Orrit, Phys. Rev. Lett. 83, 2722 (1999).

[4] J. Köhler, J. A. J. M. Disselhorst, M. C. J. M. Donckers, E. J. J. Groenen, J. Schmidt, and W. E. Moerner, Nature (London) 363, 242 (1993).

[5] J. Wrachtrup, C. von Borczyskowski, J. Bernard, M. Orrit, and R. Brown, Phys. Rev. Lett. 71, 3565 (1993).

[6] I. Gerhardt, G. Wrigge, G. Zumofen, J. Hwang, A. Renn, and V. Sandoghdar, Phys. Rev. A 79, 011402(R) (2009).

[7] R. Hildner, D. Brinks, and N. F. V. Hulst, Nat. Phys. 7, 172 (2011).

[8] J. Hwang, M. Pototschnig, R. Lettow, G. Zumofen, A. Renn, S. Götzinger, and V. Sandoghdar, Nature (London) 460, 76 (2009).
[9] Y. L. A. Rezus, S. G. Walt, R. Lettow, A. Renn, G. Zumofen, S. Götzinger, and V. Sandoghdar, Phys. Rev. Lett. 108, 093601 (2012).

[10] M. Wallquist, K. Hammerer, P. Rabl, M. Lukin, and P. Zoller, Physica Scripta 2009, 014001 (2009).

[11] S. Faez, P. Türschmann, H. R. Haakh, S. Götzinger, and V. Sandoghdar, arXiv:1407.2846 [Phys. Rev. Lett. (to be published)].

[12] P. Tamarat, A. Maali, B. Lounis, and M. Orrit, J. Phys. Chem. A 104, 1 (2000).

[13] M. Orrit, J. Bernard, A. Zumbusch, and R. I. Personov, Chem. Phys. Lett. 196, 595 (1992).

[14] F. Kulzer, R. Matzke, C. Bräuchle, and T. Basché, J. Phys. Chem. A 103, 2408 (1999).

[15] P. Bordat, M. Orrit, R. Brown, and A. Würger, Chem. Phys. 258, 63 (2000). 
[16] S. Kummer, T. Basché, and C. Bräuchle, Chem. Phys. Lett. 229, 309 (1994).

[17] J.-M. Caruge and M. Orrit, Phys. Rev. B 64, 205202 (2001).

[18] T. Plakhotnik, Chem. Phys. Chem. 7, 1699 (2006).

[19] T. Plakhotnik, J. Lumin. 127, 235 (2007).

[20] J. R. Platt, J. Chem. Phys. 22, 1448 (1954).

[21] C. Cohen-Tannoudji, J. Dupont-Roc, and G. Grynberg, AtomPhoton Interactions: Basic Processes and Applications (WileyVCH, Berlin, 1998).

[22] M. Devoret, S. Girvin, and R. Schoelkopf, Ann. Phys. 16, 767 (2007).

[23] R. A. L. Vallée, M. Van Der Auweraer, F. C. De Schryver, D. Beljonne, and M. Orrit, Chem. Phys. Chem. 6, 81 (2005).

[24] F. Dolde, H. Fedder, M. W. Doherty, T. Nöbauer, F. Rempp, G. Balasubramanian, T. Wolf, F. Reinhard, L. C. L. Hollenberg, F. Jelezko, and J. Wrachtrup, Nat. Phys. 7, 459 (2011).

[25] V. Bouchiat, D. Vion, P. Joyez, D. Esteve, and M. H. Devoret, Physica Scripta 1998, 165 (1998).

[26] G. Wrigge, I. Gerhardt, J. Hwang, G. Zumofen, and V. Sandoghdar, Nat. Phys. 4, 60 (2008).

[27] M. H. Devoret and R. J. Schoelkopf, Nature (London) 406, 1039 (2000).

[28] E. Betzig, Opt. Lett. 20, 237 (1995).

[29] C. Hettich, C. Schmitt, J. Zitzmann, S. Kühn, I. Gerhardt, and V. Sandoghdar, Science 298, 385 (2002).
[30] S. Weisenburger, B. Jing, D. Hänni, L. Reymond, B. Schuler, A. Renn, and V. Sandoghdar, Chem. Phys. Chem. 15, 763 (2014).

[31] A. A. Middleton and N. S. Wingreen, Phys. Rev. Lett. 71, 3198 (1993).

[32] C. J. Gorter, Physica 17, 777 (1951).

[33] J.-F. Dayen, E. Devid, M. V. Kamalakar, D. Golubev, C. Guédon, V. Faramarzi, B. Doudin, and S. J. van der Molen, Adv. Mater. 25, 400 (2013).

[34] S. Sadewasser and T. Glatzel, Kelvin Probe Force Microscopy: Measuring and Compensating Electrostatic Forces (Springer, Berlin, 2011).

[35] L. S. Levitov, H. Lee, and G. B. Lesovik, J. Math. Phys. 37, 4845 (1996).

[36] J. Bylander, T. Duty, and P. Delsing, Nature (London) 434, 361 (2005).

[37] A. Nicolet, M. A. Kolchenko, B. Kozankiewicz, and M. Orrit, J. Chem. Phys. 124, 164711 (2006).

[38] A. A. L. Nicolet, P. Bordat, C. Hofmann, M. A. Kol'chenko, B. Kozankiewicz, R. Brown, and M. Orrit, Chem. Phys. Chem. 8, 1929 (2007).

[39] C. Brunel, P. Tamarat, B. Lounis, J. C. Woehl, and M. Orrit, J. Phys. Chem. A 103, 2429 (1999).

[40] V. L. Colvin, K. L. Cunningham, and A. P. Alivisatos, J. Chem. Phys. 101, 7122 (1994).

[41] S. A. Empedocles and M. G. Bawendi, Science 278, 2114 (1997). 